\title{
The Effect of Origanum majorana Supplementation on Growth Performance, Blood parameters and Meat Quality in BUT9 Commercial Turkeys
}

\author{
Ahmed, E.G. and A.M. Abdel-Ghany \\ Animal Production Department, College of Agriculture, Suez Canal University, Al-Ismailia, Egypt
}

Received: $13 / 7 / 2015$

\begin{abstract}
This study was conducted to evaluate that effect of a herb marjoram (Origanum majorana L) supplementation on the growth performance, blood parameters, and meat quality in BUT9 commercial turkeys. 57 BUT9 commercial turkeys at $8 \mathrm{wk}$ of age (initial starting age with average body weight $1533 \pm 25.32$ g) were divided randomly into three treatments. Each treatment had two replicates with unequal number of birds of each treatment. A group of chicks was fed a basal diet (control) and the remaining two groups of Turkeys were fed the basal diet supplemented with marjoram at a concentration of $0.4 \%$ (T1) and $0.8 \%$ (T2), respectively, until 19th wk of age (marketing age). Results revealed that body weight and body weight gain was improved significantly $(\mathrm{P} \leq 0.05)$, feed intake was decreased and thus feed conversion was improved by feeding marjoram. These results showed that feeding on T1 diet improved non-significantly the percentage of eviscerated weight. Both creatinine and cholesterol levels of two marjoram treatments reduced significantly $(\mathrm{P} \leq 0.05)$ compared with controls. The supplementing of Origanum majorana to basal diet increased values of albumen, globulin, total protein while AST, ALT and ttriglycerides values reduced without significant. Significant treatments effects $(\mathrm{P} \leq 0.05$ or $\mathrm{P} \leq 0.01)$ were detected on tenderness of breast meat, lightness color of thigh and breast meat, redness and yellowness color of thighs only. The best values of meat quality measurements generally were recorded of two groups fed on treated diets containing marjoram compared with control group. It seemed from the results, supplementing of marjoram to basal diet improved growth performance, blood parameters, and meat quality of BUT9 turkeys.
\end{abstract}

Key words: Medicinal plants; Origanum majorana; BUT9 Turkeys; Growth performance; Meat physical traits.

\section{INTRODUCTION}

Natural feed additives of plant are safer and healthier than synthetic additives. Adding Herbs and herbal products to poultry rations as replacers of the comparable synthetic products, is assumed to instigate improvement of many productive performance.

A herb, is a plant whose leaves, seeds, or flowers are used for flavoring food or in medicine. Medicinal plant extracts were developed and proposed for use in food as natural antimicrobials (Del Campo et al., 2000 and Hsieh et al., 2001). Bacterial and fungal infections pose greater threats to health and hence the use of natural antimicrobial compounds is important in the control of human and plant diseases of microbial origin. Craig (1999) showed that a diet with culinary herbs are used generously to flavor food provides a variety of active phytochemicals that promote health and protect against chronic diseases. Leeja and Thoppil (2007) reported that the medicinal effects of marjoram on gastrointestinal tract stimulant, tonic, carminative, diaphoretic, hypoglycemic, diuretic and antibacterial. Parry (1969) described marjoram to have properties of antiseptic, antispasmodic, carminative, stimulant, expectorant and nerve tonic. Heinonen et al. (1998) showed that some natural polyphenols have therapeutic effects or a protective action against cardiovascular diseases and some cancers. Also, slowing down the ageing process and in the treatment of human diseases such as atherosclerosis and cancer reported by Tiziana and Dorman (1998). Currently, there is an increasing interest in using herbs in animal nutrition, in order to replace the use of antibiotics and ionophore anticoccidials. Herbs, Medicinal and aromatic plants are preferable as feed additives and growth promoters causing safe improvements in many growth traits (Al-
Harthi, 2002 a, b; El-Kaiaty et al., 2002 a, b; Soliman et al., 2003 and El-Mallah et al., 2005) and positive effects on immunity and viability (Soliman et al., 1995 and Soliman et al., 1999). Giannenas et al. (2005) reported that using dehydrated oregano plants improved body weight gain and feed conversion ratio when incorporated in chicken diets. Dried oregano leaves ration improved feed conversion ratio in early maturing female turkeys (Bampidis et al. 2005). Ayala et al. (2011) reported that dietary supplementation with dried oregano improved rabbit performance. Ali (2014) showed that addition of marjoram to diet improved broiler productive performance.

Origanum majorana or sweet marjoram (Origanum majorana, formerly Majorana hortensis) of the Labiatae family is indigenous to Mediterranean countries and was known to the ancient Egyptians, Greeks and Romans (Tainter and Grenis, 1993). Marjoram a common medicinal plant and contains about 0.5 to $3 \%$ oil. Hermann (1973) showed that Marjoram oil chemical composition includes borneol, terpinene, pinene, sabinene and terpineol. Also, Cowan (1999) reported that Origanum majorana are rich in a wide variety of secondary metabolites, such as terpenoids, which have been found to have antimicrobial properties. Triantaphyllou et al. (2001) gave evidence that Origanum majorana, sage and dittany extracts have a remarkable capacity in retarding lipid oxidation and showed antioxidant activity due to bound forms of phenolic compounds such as hydroxycinnamic acids and flavonoids.

The study aimed to estimate the effect of adding two levels (T1: $0.4 \%$ and T2: $0.8 \%$ ) of marjoram (Origanum majorana), to basal ration (Control) on the growth performance, blood parameters and meat quality in BUT9 Turkey. 


\section{MATERIALS AND METHODS}

This experimental was conducted in accordance with the guidelines of the department of Animal and fisheries production, faculty of Agriculture, Suez Canal University, Ismailia, Egypt, during the period from August to October 2010. Data of fifty seven BUT9, (light white and sex-identifiable-turkey strain from 8 wks of age (initial experimental starting age with average body weight $1533 \pm 25.32$ g.) till marketing (at 19 wks of age). Birds were assigned randomly into three treatments. Each treatment had two replicates with unequal number of birds and birds in each replicate were kept in a separate floor pens with dimensions $3 \mathrm{X}$ 2 meters. The experiment was conducted in open sided housing system with using fan and hood throughout the experiment. All birds were reared under the same managerial, hygienic and environmental conditions. Photoperiod was $16 \mathrm{~h}$ light and $8 \mathrm{~h}$ dark. The mean monthly minimum and maximum ambient temperature were (24.87-33.71), (23.7-32.53) and (22.4-31.76) with average relative humidity $62.18,56.46$ and 52.08 during August, September and October months, respectively. Feeds and clean fresh water were provided ad libitum.

Three different iso-caloric iso-nitrogenous experimental rations, (Table1), were used \{namely: $0 \%$ (Control); 0.4 (T1) and $0.8 \%$ (T2) of dried plants of Marjoram (Origanum majorana)\}. The approximate chemical composition of the used Origanum majorana on an air dried basis was: Moisture, $7.56 \%$; Dry matter, 92.44\%; Organic matter, $70.86 \%$; Crude protein, $13.93 \%$; Ether extract, $3.30 \%$; Crude fiber, $14.50 \%$; Ash, 21.58\% and Nitrogen free extract (NFE), 39.13\%. Feeding rations were adjusted according to the light turkey requirements stated by NRC, 1994. Experimental rations were formulated accordingly into two age stages (The first growing stage from 8 to $12 \mathrm{wk}$ and the second finishing stage from 13 to 19 wk). Composition and calculated chemical analysis of all tested experimental diets are presented in (Table 1).

Individual body weight was recorded weekly to the nearest gram, from 8 to $19 \mathrm{wk}$ of age. Gain in weight was computed, as the difference in weight between two consecutive periods. Feed intake was recorded weekly and then feed conversion was determined by dividing feed intake on gain in weight for each replicate at a certain period. At the end of the experiment, at least one bird per replicate of different treatments was slaughtered for meat physical and chemical evaluations. Organs carcass were weighed, relative organ weights were calculated on live body weight basis.

At marketing age (19 wk), individual blood samples were taken into dry clean heparinized centrifuge tubes from two birds /treatment (One male and one female), and centrifuged at $3000 \mathrm{rpm}$ for 20 min. The clear plasma samples were carefully drawn and transferred to dry, clean small glass bottles, and stored at $\leq-20^{\circ} \mathrm{C}$ in a deep freezer until the time of chemical determination. Evaluated biochemical characteristic of blood plasma encompassed the total cholesterol, g/100 ml according to Richmond (1973); Triglycerides, mg/100 ml (Bernd and George, 1977); Creatinine, $\mathrm{mg} / 100 \mathrm{ml}$ (Bartels et al., 1972); Total protein, g/100 $\mathrm{ml}$ according to Weichselbaum (1946) and Albumin, g/100 ml (Doumas et al., 1971). Globulin concentration was calculated as the difference between total protein and albumin concentrations.

Table (1): Determined chemical compositions of experimental rations ( $0.0 \%$ : Control, $0.40 \%$ : T1 and $0.80 \%$ :T2) of Origanum majorana during the growing (till 12wk) and finishing (from 13 till 19 wk of age) stages.

\begin{tabular}{|c|c|c|c|c|c|c|}
\hline \multirow{2}{*}{ Ingredient } & \multicolumn{3}{|c|}{ First stage (grower) } & \multicolumn{3}{|c|}{ Second stage (Finisher) } \\
\hline & Control & T1 & $\mathbf{T 2}$ & Control & $\mathbf{T 1}$ & $\mathbf{T 2}$ \\
\hline Yellow corn & 61.60 & 61.20 & 60.80 & 68.80 & 68.40 & 68.00 \\
\hline Soya bean (44 \%) & 26.00 & 26.00 & 26.00 & 18.50 & 18.50 & 18.50 \\
\hline Broiler concentrate & 10.00 & 10.00 & 10.00 & 10.00 & 10.00 & 10.00 \\
\hline Animal fat & 1.50 & 1.50 & 1.50 & 2.00 & 2.00 & 2.00 \\
\hline Di-Calcium phosphate & 0.30 & 0.30 & 0.30 & 0.10 & 0.10 & 0.10 \\
\hline $\mathrm{NaCl}$ & 0.50 & 0.50 & 0.50 & 0.50 & 0.50 & 0.50 \\
\hline Dl-Methionine & 0.10 & 0.10 & 0.10 & 0.10 & 0.10 & 0.10 \\
\hline Marjoram & 0.00 & 0.40 & 0.80 & 0.00 & 0.40 & 0.80 \\
\hline \multicolumn{7}{|l|}{ Determined analysis } \\
\hline Crude protein & 21.62 & 21.24 & 21.50 & 18.86 & 18.93 & 19.15 \\
\hline Moisture & 10.52 & 10.40 & 10.28 & 10.22 & 10.17 & 10.54 \\
\hline Ether extract & 2.57 & 2.77 & 2.82 & 2.65 & 2.73 & 2.75 \\
\hline Crude fiber & 3.22 & 3.36 & 3.58 & 3.15 & 3.26 & 3.55 \\
\hline Ash & 6.44 & 6.58 & 6.54 & 6.18 & 6.27 & 6.34 \\
\hline $\mathrm{ME}(\mathrm{Kcal} / \mathrm{kg})$ & 3025 & 3014 & 3002 & 3143 & 3132 & 3120 \\
\hline
\end{tabular}

(vitamin and minerals mixture premix was added at the ratio of $3 \mathrm{~kg}$ per ton as a part of the Broiler concentrate): Each $3 \mathrm{~kg}$ of vitamin and minerals mixture contained 12,000,000 IU Vit. A; 2,000,000 IU Vit. $\mathrm{D}_{3} ; 10,000 \mathrm{mg}$ Vit. E; 2,000 mg Vit. $\mathrm{K}_{3} ; 1,000 \mathrm{mg}$ Vit. $\mathrm{B}_{1} ; 5,000 \mathrm{mg}$ Vit. $\mathrm{B}_{2} ; 1,500 \mathrm{mg}$ Vit. $\mathrm{B}_{6} ; 10 \mathrm{mg}$ Vit. $\mathrm{B}_{12} ; 10,000 \mathrm{mg}$ pantothenic acid; 30,000 mg Nicotinic acid, 1,000 mg Folic acid; $50 \mathrm{mg}$ Biotin; $250,000 \mathrm{mg}$ choline chloride, $10,000 \mathrm{mg} \mathrm{Cu}, 1,000 \mathrm{mg} \mathrm{I} ; 30,000 \mathrm{mg} \mathrm{Fe}$; 50,000 mg Zn, 60,000 mg Mn, $100 \mathrm{mg}$ Co and $100 \mathrm{mg}$ Se. 
In addition, Alanine transaminase (ALT U/L, Formerly sGPT), Aspartate transaminase (AST U/L, Formerly sGOT) were determined according to Tietz (1995). These entire biochemical characteristic were colorimetrically determined using commercial kits purchased from the Egyptian American Company of Laboratory Services and El-Nasr Pharmaceuticals Chemical Company, Cairo, Egypt.

Six BUT9 birds (three from each sex) at the $19^{\text {th }}$ wk. of age were randomly selected and scarified to obtain meat physical (i.e. color intensity, $\mathrm{pH}$, water holding capacity and tenderness for thigh and breast muscles samples, measured directly after slaughter) and chemical (i.e. uric acid in blood plasma, liver as well as thigh and breast muscles, were enzymatically determined according to Kageyama (1971) after a period of preserving in a refrigerator) characteristics. Tenderness and water holding capacity (WHC) were determined according to Jauregui et al. (1981); pH according to Jeacocke (1977) and color intensity according to (CIE - International Commission on Illumination, 1978).

Data of BUT9 turkeys were analyzed using general linear model procedure of SAS software (SAS Institute, 1998). Difference among treatment means or between sexes were tested for significances using Duncan's Multiple Range Test (Duncan, 1955).

\section{RESULTS AND DISCUSSION}

\section{Body weight and weight gain}

Body weight differences due to various treatments were significant $(\mathrm{P} \leq 0.05)$ at $12,13,14,15$ and 16 wk of age, as well as gain in weight at 11-12 and 8-19 wk of age (Tables 2 and 3). For the two trait classes, the highest values of treatment means were generally for $\mathrm{T} 2$, followed in most cases by $\mathrm{T} 1$ then the control group (Tables 2 and 3). In this respect, Abdo et al. (2003), Soliman et al. (2003) with broiler and Seleem et al. (2007) with growing New Zealand rabbits indicated that body weight and gain in weight improved significantly by various feeding substitutes with Origanum majorana. Mona Osman et al. (2010) and Ali (2014) showed that supplementing marjoram leaves powder to broiler diet improved significantly body weight and weight gain at most ages studied. Also, Giannenas et al. (2005) and Florou-Paneri et al. (2006) reported that using oregano plants improved body weight gain when incorporated in chicken diets.

These progressive positive effects of marjoram on body and gain weight could be ascribed to the positive effects on immunity, Craig (1999); antifungal activity, Tiziana and Dorman (1998), antibacterial properties, Yadava and Saini (1991), wide variety of secondary metabolites such as terpenoids, Cowan (1999) and antioxidant activity, Triantaphyllou et al. (2001). Guo (2003) showed that medicinal plants components mechanism may include improving the physical conditions of gut ecosystem and enhancing function of immune system of chickens.

Sex differences on body weight and gain in weight were generally significant $(\mathrm{P} \leq 0.05 ; \mathrm{P} \leq 0.01 ; \mathrm{P} \leq 0.001$; $\mathrm{P} \leq 0.0001)$ at most ages for body weight, throughout the whole age intervals and those intervals from 8-9, 10-11 and 11-12 wk for gain in weight (Tables 2 and 3). BUT9 turkey toms were superior to females in body weight and gain in weight at all ages considered. The difference in values between males and females increased progressively with advance of age till marketing for body weight and throughout the intervals from 8-14 wk and declined subsequently till age of marketing for gain in weight.

Similar results were also obtained by Mostafa and Nofal (2000), Isguzar (2003), Ersoy and Çelik (2007), Zaky and Amin (2007) and Ilori et al. (2010) with different breeds. Zaky and Amin (2007) demonstrated significant sex differences in turkeys' body weight (Bronze vs. Baladi) at all ages from 4 to 20 wks of age along with males were heavier than females. Also, Significant sex differences with males being heavier for body weight at different ages and from 20 to 24 wk of ages for two acclimatized breeds of turkey (Broad Breasted Bronze, White Holland and their reciprocal crosses) reported by Mostafa and Nofal (2000). This substantial body weight performance of turkey males, versus females, is expected to be hormonal mediated which elaborates and amplifies as approaching puberty. In addition, the presence of such sexual dimorphism in favor of males in the growth performance can be partially attributed to aggressiveness and dominance of males when are not reared separately (Hancock et al. 1994).

\section{Feed intake and conversion}

Treatments affected feed intake significantly $(\mathrm{P} \leq$ $0.05 ; \mathrm{P} \leq 0.01$ or $\mathrm{P} \leq 0.001)$ at the 9,17 and $19 \mathrm{wk}$ of age as well as during the age intervals from 9-19 wk of age. Apart of treatments effect, feed intake increases with advance of age (Table 4). During the periods from 16 to $19 \mathrm{wk}$. and from 9 to $19 \mathrm{wk}$ of age, the lowest feed intake values were recorded by T1, control and T2, respectively. Soliman et al. (2003) with broiler and Seleem et al. (2007) with New Zealand White rabbits reported that total feed intake values reduced insignificantly in contrast with the marjoram untreated group. Mona Osman et al. (2010) and Ali (2014) reported that significant decrease of feed intake with addition of marjoram to broiler diet during most the age periods.

Non-significant treatment effects on feed conversion were detected during all studied intervals from 9 to $19 \mathrm{wk}$. (Table 5). Apart from significance, a trend was detected for $\mathrm{T} 1$ to give the best (lowest), values of feed conversion followed by $\mathrm{T} 2$ and finally control treatment. In this respect, Abdo et al. (2003), Soliman et al. (2003), Mona Osman et al. (2010) and Ali (2014) with broiler and Seleem et al. (2007) with New Zealand White rabbits found conversion improvement for fed diets containing marjoram versus control. Giannenas et al. (2005) and Florou-Paneri et al. (2006) reported that using oregano plants improved feed conversion ratio when incorporated in chicken diets. Dried oregano leaves at $1.25,2.5$, and $3.75 \mathrm{~g} / \mathrm{kg}$ ration improved feed conversion ratio in early maturing female turkeys (Bampidis et al., 2005). The improvement in feed intake and conversion may be due to the ability of 
marjoram to increase the efficiency of digestion by increasing digestive enzymes, curing or preventing basic intestinal infections and relieving diarrhea (Hallent, 2015). Cowan (1999) concluded that the positive effects of Origanum majorana may be due to abundance and wide variety of secondary metabolites, such as terpenoids, which have been found to have antimicrobial properties.

\section{Slaughter parameters}

Differences in slaughter parameters due to treatments and sex effects were non-significant (Table 6). These results showed that Tldiet improved the percentage of eviscerated weight; but the percentage of eviscerated weight of male was better than female.
These results agreement with Bampidis et al. (2005) who reported no significant effect of oregano content in diet on carcass weights, carcass yield, and the relative weights of the heart and liver of early maturing female turkeys. On another hand, Seleem et al. (2007) reported that supplementing Origanum majorana to growing diets of New Zealand White rabbits significantly increased dressing percentage by $6.1 \% .0$. The improvement in carcass traits for treated diet with marjoram may be related with the increase in body weight of birds. Isguzar (2003), Ersoy and Çelik (2007) and Shamseldin et al. (2014) evidenced significant effects of sex on hot carcass of turkeys where males were better than females.

Table (2): Least square means, standard errors (S.E) and coefficients of variation (CV\%) for body weight (g) of BUT9 turkeys at different ages fed on diets contained 0.0 (control), $0.4 \%$ (T1) or $0.8 \%$ (T2) Origanum majorana.

\begin{tabular}{|c|c|c|c|c|c|c|c|c|c|}
\hline $\begin{array}{l}\text { Age } \\
\text { (wk) }\end{array}$ & & Control & T1 & $\mathbf{T} 2$ & Sig. & Male & Female & Sig & $\begin{array}{c}\text { Overall } \\
\text { mean }\end{array}$ \\
\hline \multirow{3}{*}{$8 \dagger$} & Mean & 1534.69 & 1534.55 & 1532.11 & \multirow{3}{*}{ NS } & 1613.17 & 1445.56 & \multirow{3}{*}{ NS } & 1533.77 \\
\hline & SE & 40.09 & 63.98 & 59.11 & & 39.11 & 49.82 & & 32.98 \\
\hline & CV\% & 10.45 & 19.56 & 16.82 & & 13.28 & 17.91 & & 16.23 \\
\hline \multirow{3}{*}{9} & Mean & 1954.06 & 1905.91 & 1970.00 & \multirow{3}{*}{ NS } & 2037.50 & 1833.33 & \multirow{3}{*}{ NS } & 1940.79 \\
\hline & $\mathrm{SE}$ & 53.06 & 83.30 & 71.21 & & 47.61 & 66.42 & & 42.11 \\
\hline & CV\% & 10.86 & 20.50 & 15.76 & & 12.80 & 18.83 & & 16.38 \\
\hline \multirow{3}{*}{10} & Mean & 2453.75 & 2451.82 & 2503.68 & \multirow{3}{*}{ NS } & 2594.33 & 2331.11 & \multirow{3}{*}{ NS } & 2469.65 \\
\hline & SE & 54.87 & 95.03 & 80.11 & & 60.32 & 65.08 & & 47.24 \\
\hline & $\mathrm{CV} \%$ & 8.94 & 18.18 & 13.95 & & 12.73 & 14.51 & & 14.44 \\
\hline \multirow{3}{*}{11} & Mean & 2917.19 & 2975.23 & 3007.89 & \multirow{3}{*}{ NS } & 3136.67 & 2784.44 & \multirow{3}{*}{$*$} & 2969.82 \\
\hline & SE & 70.17 & 110.36 & 100.70 & & 72.98 & 75.04 & & 56.94 \\
\hline & $\mathrm{CV} \%$ & 9.62 & 17.40 & 14.59 & & 12.74 & 14.00 & & 14.48 \\
\hline \multirow{3}{*}{12} & Mean & $3420.63^{b}$ & $3580.91^{\mathbf{a b}}$ & $3660.00^{\mathrm{a}}$ & \multirow{6}{*}{$*$} & 3781.67 & 3318.52 & \multirow{3}{*}{$* *$} & 3562.28 \\
\hline & SE & 77.22 & 119.77 & 139.76 & & 79.57 & 97.63 & & 69.11 \\
\hline & $\mathrm{CV} \%$ & 9.03 & 15.69 & 16.64 & & 11.53 & 15.29 & & 14.65 \\
\hline \multirow{3}{*}{13} & Mean & $4088.13^{b}$ & $4260.00^{\mathbf{a b}}$ & $4348.95^{\mathrm{a}}$ & & 4547.00 & 3901.85 & \multirow{3}{*}{$* * *$} & 4241.40 \\
\hline & SE & 92.35 & 143.43 & 166.49 & & 92.24 & 108.84 & & 82.33 \\
\hline & CV\% & 9.04 & 15.79 & 16.69 & & 11.11 & 14.49 & & 14.65 \\
\hline \multirow{3}{*}{14} & Mean & $4886.88^{b}$ & $5044.55^{\mathbf{a b}}$ & $5198.42^{a}$ & \multirow{3}{*}{$*$} & 5437.00 & 4623.33 & \multirow{3}{*}{$\begin{array}{c}* * * \\
*\end{array}$} & 5051.58 \\
\hline & SE & 108.01 & 152.21 & 194.35 & & 88.85 & 125.11 & & 92.42 \\
\hline & CV\% & 8.84 & 14.15 & 16.30 & & 8.95 & 14.06 & & 13.81 \\
\hline \multirow{3}{*}{15} & Mean & $5560.63^{b}$ & $5724.55^{\mathrm{ab}}$ & $5916.32^{a}$ & \multirow{3}{*}{$*$} & 6194.00 & 5240.74 & \multirow{3}{*}{$\begin{array}{c}* * * \\
*\end{array}$} & 5742.46 \\
\hline & SE & 122.24 & 164.52 & 217.19 & & 95.47 & 133.40 & & 102.19 \\
\hline & CV\% & 8.79 & 13.48 & 16.00 & & 8.44 & 13.23 & & 13.44 \\
\hline \multirow{3}{*}{16} & Mean & $6198.75^{b}$ & $6360.91^{\mathbf{a b}}$ & $6566.05^{\mathrm{a}}$ & \multirow{3}{*}{$*$} & 6926.17 & 5781.11 & & 6383.77 \\
\hline & $\mathrm{SE}$ & 145.05 & 182.66 & 240.34 & & 111.06 & 131.00 & $* * *$ & 113.90 \\
\hline & CV\% & 9.36 & 13.47 & 15.96 & & 8.78 & 11.77 & $*$ & 13.47 \\
\hline \multirow{3}{*}{17} & Mean & 6863.13 & 6962.27 & 7246.32 & & 7615.00 & 6378.15 & & 7029.12 \\
\hline & SE & 159.26 & 191.03 & 259.31 & NS & 122.73 & 133.62 & $*$ & 121.83 \\
\hline & CV\% & 9.28 & 12.87 & 15.60 & & 8.83 & 10.89 & & 13.09 \\
\hline & Mean & $7351.88^{\mathbf{b}}$ & $7569.55^{\mathbf{a b}}$ & $7847.37^{\mathbf{a}}$ & & 8245.67 & 6884.81 & & 7601.05 \\
\hline 18 & SE & 185.76 & 212.83 & 283.91 & NS & 147.87 & 139.21 & $*$ & 135.89 \\
\hline & $\mathrm{CV} \%$ & 10.11 & 13.19 & 15.77 & & 9.82 & 10.51 & & 13.50 \\
\hline & Mean & $7861.25^{b}$ & $8045.00^{\mathbf{a b}}$ & $8394.21^{\mathrm{a}}$ & & 8801.00 & 7341.85 & & 8109.82 \\
\hline 19 & SE & 188.90 & 220.92 & 307.88 & NS & 160.05 & 139.23 & $*$ & 143.96 \\
\hline & $\mathrm{CV} \%$ & 9.61 & 12.88 & 15.99 & & 9.96 & 9.85 & & 13.40 \\
\hline
\end{tabular}

$8 \dagger=$ Initial body weight at $8 \mathrm{wk}$ of age

$a, b, \ldots$ Treatment means having different superscript litters within the same raw are significantly different at $(P \leq 0.05)$ using Duncan's Multiple Range test. 
Table (3): Least square means, standard errors (S.E.) and coefficients of variation (CV\%) for gain in weight (g) of BUT9 turkeys at different ages fed on diets contained 0.0 (control), $0.4 \%$ (T1) or 0.8\% (T2) Origanum majorana.

\begin{tabular}{|c|c|c|c|c|c|c|c|c|c|}
\hline $\begin{array}{l}\text { Period } \\
(\mathbf{W k})\end{array}$ & & Control & T1 & $\mathbf{T} 2$ & Sig. & Male & Female & Sig & $\begin{array}{c}\text { Overall } \\
\text { mean }\end{array}$ \\
\hline 8-9 & $\begin{array}{l}\text { Mean } \\
\text { SE } \\
\text { CV }\end{array}$ & $\begin{array}{r}419.38 \\
32.44 \\
30.94\end{array}$ & $\begin{array}{r}371.36 \\
28.30 \\
35.75\end{array}$ & $\begin{array}{r}437.89 \\
29.65 \\
29.52\end{array}$ & NS & $\begin{array}{c}424.33 \\
23.54 \\
30.38\end{array}$ & $\begin{array}{c}387.78 \\
25.90 \\
34.70\end{array}$ & NS & $\begin{array}{c}407.02 \\
17.45 \\
32.37\end{array}$ \\
\hline $9-10$ & $\begin{array}{l}\text { Mean } \\
\text { SE } \\
\text { CV }\end{array}$ & $\begin{array}{r}499.69 \\
20.36 \\
16.30\end{array}$ & $\begin{array}{r}545.91 \\
26.75 \\
22.98\end{array}$ & $\begin{array}{r}533.68 \\
22.55 \\
18.41\end{array}$ & NS & $\begin{array}{c}556.83 \\
18.84 \\
18.54\end{array}$ & $\begin{array}{c}497.78 \\
19.46 \\
20.32\end{array}$ & NS & $\begin{array}{c}528.86 \\
13.99 \\
19.97\end{array}$ \\
\hline $10-11$ & $\begin{array}{l}\text { Mean } \\
\text { SE } \\
\text { CV }\end{array}$ & $\begin{array}{r}463.44 \\
26.70 \\
23.04\end{array}$ & $\begin{array}{r}523.41 \\
28.53 \\
25.57\end{array}$ & $\begin{array}{r}504.21 \\
31.07 \\
26.86\end{array}$ & NS & $\begin{array}{c}542.33 \\
22.76 \\
22.99\end{array}$ & $\begin{array}{c}453.33 \\
22.25 \\
25.50\end{array}$ & * & $\begin{array}{c}500.18 \\
16.89 \\
25.50\end{array}$ \\
\hline 11-12 & $\begin{array}{l}\text { Mean } \\
\text { SE } \\
\text { CV }\end{array}$ & $\begin{array}{r}503.44^{\mathbf{b}} \\
26.68 \\
21.20\end{array}$ & $\begin{array}{c}605.68^{\mathbf{a b}} \\
37.93 \\
29.38\end{array}$ & $\begin{array}{c}652.11^{\mathrm{a}} \\
58.54 \\
39.13\end{array}$ & * & $\begin{array}{c}645.00 \\
34.26 \\
29.09\end{array}$ & $\begin{array}{c}534.07 \\
37.93 \\
36.90\end{array}$ & NS & $\begin{array}{c}592.46 \\
26.29 \\
33.50\end{array}$ \\
\hline $12-13$ & $\begin{array}{l}\text { Mean } \\
\text { SE } \\
\text { CV }\end{array}$ & $\begin{array}{r}667.50 \\
37.48 \\
22.46\end{array}$ & $\begin{array}{r}679.09 \\
41.50 \\
28.66\end{array}$ & $\begin{array}{r}688.95 \\
36.30 \\
22.96\end{array}$ & NS & $\begin{array}{c}765.33 \\
27.14 \\
19.42\end{array}$ & $\begin{array}{c}583.33 \\
26.03 \\
23.19\end{array}$ & $* * *$ & $\begin{array}{c}679.12 \\
22.30 \\
24.79\end{array}$ \\
\hline $13-14$ & $\begin{array}{l}\text { Mean } \\
\text { SE } \\
\text { CV }\end{array}$ & $\begin{array}{r}798.75 \\
48.44 \\
24.26\end{array}$ & $\begin{array}{r}784.55 \\
28.99 \\
17.33\end{array}$ & $\begin{array}{r}849.47 \\
45.21 \\
23.20\end{array}$ & NS & $\begin{array}{c}890.00 \\
27.51 \\
16.93\end{array}$ & $\begin{array}{c}721.48 \\
30.06 \\
21.65\end{array}$ & $\begin{array}{c}* * * \\
*\end{array}$ & $\begin{array}{c}810.18 \\
23.05 \\
21.48\end{array}$ \\
\hline $14-15$ & $\begin{array}{l}\text { Mean } \\
\text { SE } \\
\text { CV }\end{array}$ & $\begin{array}{r}673.75 \\
44.14 \\
26.20\end{array}$ & $\begin{array}{r}680.00 \\
39.56 \\
27.29\end{array}$ & $\begin{array}{r}717.89 \\
35.45 \\
21.52\end{array}$ & NS & $\begin{array}{c}757.00 \\
29.27 \\
21.18\end{array}$ & $\begin{array}{c}617.41 \\
29.71 \\
25.00\end{array}$ & $* * *$ & $\begin{array}{c}690.88 \\
22.68 \\
24.78\end{array}$ \\
\hline $15-16$ & $\begin{array}{l}\text { Mean } \\
\text { SE } \\
\text { CV }\end{array}$ & $\begin{array}{r}638.13 \\
47.47 \\
29.76\end{array}$ & $\begin{array}{r}636.36 \\
49.70 \\
36.63\end{array}$ & $\begin{array}{r}649.74 \\
40.20 \\
26.97\end{array}$ & NS & $\begin{array}{c}732.17 \\
36.87 \\
27.58\end{array}$ & $\begin{array}{c}540.37 \\
27.52 \\
26.46\end{array}$ & $* * *$ & $\begin{array}{c}641.32 \\
26.47 \\
31.16\end{array}$ \\
\hline $16-17$ & $\begin{array}{l}\text { Mean } \\
\text { SE } \\
\text { CV }\end{array}$ & $\begin{array}{r}664.38 \\
55.52 \\
33.43\end{array}$ & $\begin{array}{r}601.36 \\
32.62 \\
25.44\end{array}$ & $\begin{array}{r}680.26 \\
37.63 \\
24.11\end{array}$ & NS & $\begin{array}{c}688.83 \\
34.82 \\
27.69\end{array}$ & $\begin{array}{c}540.37 \\
27.52 \\
26.46\end{array}$ & $*$ & $\begin{array}{c}645.35 \\
23.66 \\
27.68\end{array}$ \\
\hline 17-18 & $\begin{array}{l}\text { Mean } \\
\text { SE } \\
\text { CV }\end{array}$ & $\begin{array}{r}488.75 \\
48.81 \\
39.95\end{array}$ & $\begin{array}{r}607.27 \\
50.94 \\
39.34\end{array}$ & $\begin{array}{r}601.05 \\
41.62 \\
30.18\end{array}$ & NS & $\begin{array}{c}630.67 \\
46.98 \\
40.81\end{array}$ & $\begin{array}{c}506.67 \\
23.20 \\
23.80\end{array}$ & $* *$ & $\begin{array}{c}571.93 \\
28.08 \\
37.07\end{array}$ \\
\hline 18-19 & $\begin{array}{l}\text { Mean } \\
\text { SE } \\
\text { CV }\end{array}$ & $\begin{array}{r}509.38 \\
46.52 \\
36.53\end{array}$ & $\begin{array}{r}475.45 \\
32.67 \\
32.23\end{array}$ & $\begin{array}{r}546.84 \\
46.02 \\
36.69\end{array}$ & NS & $\begin{array}{c}555.33 \\
36.73 \\
36.23\end{array}$ & $\begin{array}{c}457.04 \\
26.09 \\
29.66\end{array}$ & $* *$ & $\begin{array}{c}508.77 \\
23.67 \\
35.13\end{array}$ \\
\hline 8-19 & $\begin{array}{c}\text { Mean } \\
\text { SE } \\
\text { CV }\end{array}$ & $\begin{array}{r}6326.56^{\mathbf{b}} \\
210.76 \\
13.33\end{array}$ & $\begin{array}{c}6510.45^{\mathrm{ab}} \\
185.60 \\
13.37\end{array}$ & $\begin{array}{r}6862.11^{\mathrm{a}} \\
275.40 \\
17.49\end{array}$ & $*$ & $\begin{array}{c}7187.83 \\
155.72 \\
11.87\end{array}$ & $\begin{array}{c}5896.30 \\
121.47 \\
10.70\end{array}$ & $\begin{array}{c}* * * \\
*\end{array}$ & $\begin{array}{c}6576.05 \\
131.45 \\
15.09\end{array}$ \\
\hline
\end{tabular}

$\mathrm{a}, \mathrm{b}, \ldots$ Treatment means having different superscript litters within the same raw are significantly different at $(\mathrm{P} \leq 0.05)$ using Duncan's Multiple Range test.

\section{Plasma constituents}

Results revealed that both cholesterol and creatinine levels in two Origanum treatments were significantly reduced while triglycerides were non significantly reduced compared with controls (Table 7). The reduction in circulating cholesterol by Origanum can be attributed to its high unsaturated fatty acids' contents which may stimulate the cholesterol excretion through the intestine and/or enhance oxidation of cholesterol to bile salts and/or to the affluent presence of isoflavones which prevent intestinal absorption of cholesterol by competition for its absorption sites. From another hand, Mona Osman et al. (2010) and Ali (2014) reported that no significant effect of broiler marjoram diet on cholesterol, triglycerides and creatinine.
Also, Dina EL Bushuty and Naglaa, Shanshan (2012) reported that rat males fed with marjoram at 5 and $10 \%$ showed a significant decrease in the values of serum cholesterol, triglyceride and low density lipoprotein-cholesterol $(\mathrm{P}<0.05)$ but showed a significant increase in the values of serum in high density lipoprotein-cholesterol $(\mathrm{P}<0.05)$ in comparing with control rat group. Also, Nagm (2002) found that feeding with marjoram extract has led to significantly lowering triglyceride serum levels for treated rats $(p<0.01)$ compared with control. Rang and Dale (1991) reported that the hypocholesterolemic effect of marjoram can be attributed to presence of isoflavones which prevent intestinal absorption of cholesterol by competition for its absorption sites. 
Table (4): Least square means, standard errors (S.E.) and coefficients of variation (CV\%) for feed intake (g) of BUT9 turkeys at different ages fed on diets contained 0.0 (control), $0.4 \%$ (T1) or $0.8 \%$ (T2) Origanum majorana.

\begin{tabular}{|c|c|c|c|c|c|c|}
\hline Age (wk) & & Control & T1 & $\mathrm{T} 2$ & Sig. & Overall mean \\
\hline & Mean & $755.00^{\mathrm{a}}$ & $685.00^{b}$ & $712.00^{\mathrm{ab}}$ & & 717.33 \\
\hline \multirow[t]{3}{*}{9} & $\mathrm{SE}$ & 5.00 & 5.00 & 18.00 & * & 13.82 \\
\hline & $\mathrm{CV}$ & 0.93 & 1.03 & 3.57 & & 4.72 \\
\hline & Mean & 904.00 & 882.00 & 895.50 & & 893.83 \\
\hline \multirow[t]{3}{*}{10} & SE & 14.00 & 9.00 & 15.50 & NS & 7.13 \\
\hline & $\mathrm{CV}$ & 2.19 & 1.44 & 2.44 & & 1.95 \\
\hline & Mean & 940.00 & 990.00 & 986.50 & & 972.16 \\
\hline \multirow[t]{3}{*}{11} & SE & 20.00 & 80.00 & 86.50 & NS & 32.49 \\
\hline & $\mathrm{CV}$ & 3.00 & 11.42 & 12.40 & & 8.18 \\
\hline & Mean & 1170.00 & 1207.00 & 1275.50 & & 1217.50 \\
\hline \multirow[t]{3}{*}{12} & $\mathrm{SE}$ & 70.00 & 43.00 & 174.50 & NS & 53.49 \\
\hline & $\mathrm{CV}$ & 8.46 & 5.03 & 19.34 & & 10.76 \\
\hline & Mean & 1315.50 & 1245.50 & 1308.00 & & 1289.66 \\
\hline \multirow[t]{3}{*}{13} & $\mathrm{SE}$ & 25.50 & 9.50 & 22.00 & NS & 16.69 \\
\hline & $\mathrm{CV}$ & 2.74 & 1.07 & 2.37 & & 3.17 \\
\hline & Mean & 1515.00 & 1459.00 & 1560.00 & & 1511.33 \\
\hline \multirow[t]{3}{*}{14} & SE & 25.00 & 14.00 & 90.00 & NS & 30.59 \\
\hline & $\mathrm{CV}$ & 2.33 & 1.35 & 8.15 & & 4.95 \\
\hline & Mean & 1489.00 & 1513.50 & 1631.50 & & 1544.66 \\
\hline \multirow{3}{*}{15} & SE & 71.00 & 13.50 & 73.50 & NS & 38.50 \\
\hline & $\mathrm{CV}$ & 6.74 & 1.26 & 6.37 & & 6.10 \\
\hline & Mean & 1700.00 & 1620.50 & 1831.00 & & 1717.16 \\
\hline \multirow[t]{3}{*}{16} & $\mathrm{SE}$ & 50.00 & 70.50 & 72.00 & NS & 48.47 \\
\hline & $\mathrm{CV}$ & 4.15 & 6.15 & 5.56 & & 6.91 \\
\hline & Mean & $1920.00^{\mathbf{a b}}$ & $1792.50^{\mathbf{b}}$ & $2077.50^{\mathrm{a}}$ & & 1930.00 \\
\hline \multirow[t]{3}{*}{17} & SE & 30.00 & 62.50 & 22.50 & * & 55.42 \\
\hline & $\mathrm{CV}$ & 2.21 & 4.93 & 1.53 & & 7.03 \\
\hline & Mean & 2041.50 & 2031.50 & 2239.00 & & 2104.00 \\
\hline \multirow[t]{3}{*}{18} & SE & 118.50 & 13.50 & 71.00 & NS & 55.76 \\
\hline & $\mathrm{CV}$ & 8.20 & 0.94 & 4.48 & & 6.49 \\
\hline & Mean & $2241.50^{\mathbf{a b}}$ & $2038.50^{\mathbf{b}}$ & $2400.00^{\mathrm{a}}$ & & 2226.66 \\
\hline \multirow[t]{3}{*}{19} & $\mathrm{SE}$ & 18.50 & 88.50 & 10.00 & $*$ & 70.21 \\
\hline & $\mathrm{CV}$ & 1.16 & 6.14 & 0.58 & & 7.72 \\
\hline & Mean & $15992.00^{\mathbf{b}}$ & $15465.00 \mathbf{c}$ & $16917.00^{\mathrm{a}}$ & & 2226.66 \\
\hline \multirow[t]{2}{*}{$9-19$} & $\mathrm{SE}$ & 78.00 & 72.00 & 145.00 & $* *$ & 70.21 \\
\hline & $\mathrm{CV}$ & 0.69 & 0.65 & 1.21 & & 7.72 \\
\hline
\end{tabular}

$\mathrm{a}, \mathrm{b}, \ldots$ Treatment means having different superscript litters within the same raw are significantly different at $(\mathrm{P} \leq 0.05)$ using Duncan's Multiple Range test.

Birds fed on the control diet has probably acquired the highest levels (not supported by significance) of ALT and AST levels compared with two Origanum treated groups. In this respect, Dina EL Bushuty and Naglaa, Shanshan (2012) showed that the values of serum ALT, AST were significantly decreased in treated rat groups with marjoram $(\mathrm{P} \leq 0.05)$ compared with control rat group. From another hand, Seleem et al. (2007) reported that supplementing 3\% Origanum majorana to growing diets of New Zealand White rabbits had higher values of ALT and AST. Also, Mona Osman et al. (2010) with broiler reported that increase no significantly of ALT and AST levels related with fed on marjoram diet.

EL-Ashmawy et al. (2005) reported that the reduction in serum levels of aminotransferases enzymes (ALT, AST) may be due to the presence of isoflavones, polyphenols and other antioxidants in herbal plants. Also, Rodriguez-Meizoso et al. (2006) demonstrated that the effect of administration of marjoram (volatile oil, alcoholic and aqueous) induced a significant decrease in serum activities of aminotransferases enzymes.

The results showed that $\mathrm{T} 1$ but not $\mathrm{T} 2$, revealed the highest levels of albumen, globulin, and total protein and that indicate better immunity or immune enhancement for this treatment which may be due to better balance between albumen and globulin values and the higher concentration of globulin value. In this respect, Also, Seleem et al. (2007) reported that supplementing 3\% Origanum majorana to growing diets of New Zealand White rabbits, had higher blood parameters (total protein, albumin, globulin and $\mathrm{A} / \mathrm{G}$ ratio). From another hand, Mona Osman et al. (2010) reported that increase significant of globulin and decrease significant of albumin and $\mathrm{A} / \mathrm{G}$ ratio for broiler marjoram diet compared with control treatment. However, Ali (2014) showed that supplementing marjoram of broiler diet reduce significantly albumin, 
globulin and $\mathrm{A} / \mathrm{G}$ ratio than the control group and did not have any effect on total protein levels.

Some studies on different poultry species (turkey, broiler, layers and Japanese quail) reported that adding medicinal, aromatic plants or herbal additive compounds to control diets significantly decreased serum cholesterol and/or triglycerides (Abdel-Azeem, 2002; and Osman et al., 2004; El-Mallah et al., 2005) increased total protein, albumin and globulin by (Azouz, 2001); and no adverse effects on ALT, AST and creatinine by Abdel-Azeem (2002), Abdo et al. (2003), Al-Harthi (2004) and Tollba et al. (2005). Mahdi et al. (2015) reported that adding some herbal plant to the turkey diets decreasing the AST and ALT levels while showed that no differences were detected in cholesterol, total protein and creatinine. However, Emam (2006) reported that females of Japanese quail when fed medicinal and aromatic plants had higher serum cholesterol, triglycerides, total protein and globulin.

Sex effects were generally non-significant, though apparently males showed higher values of albumen, globulin, total protein, ALT, cholesterol and triglycerides while females surpassed males in $\mathrm{A} / \mathrm{G}$ ratio and AST. These results agreement with Ogundu Uduak et al. (2013) who reported that non-significant effect of sex on total protein, albumen, globulin, cholesterol, ALT, and AST in turkeys. Ghazalah et al. (1994) reported that the numeric variations in total protein, albumin and globulin could be interpreted due to many factors such as genetic, age, sex, physiological status, rearing condition, feeding as well as pathological factors.

Table (5): Least square means, standard errors (S.E.) and coefficients of variation (CV\%) for feed conversion of BUT9 turkeys at different ages fed on diets contained 0.0 (control), $0.4 \%$ (T1) or $0.8 \%$ (T2) Origanum majorana.

\begin{tabular}{|c|c|c|c|c|c|c|}
\hline Age (wk) & & Control & T1 & $\mathbf{T} 2$ & Sig. & Overall mean \\
\hline \multirow{3}{*}{9} & Mean & $1.79 \mathbf{a b}$ & $1.84 \mathbf{a}$ & $1.62 \mathbf{b}$ & \multirow{3}{*}{$\mathrm{NS}$} & 1.75 \\
\hline & SE & 0.05 & 0.03 & 0.03 & & 0.04 \\
\hline & $\mathrm{CV}$ & 3.95 & 2.68 & 3.04 & & 6.36 \\
\hline \multirow{3}{*}{10} & Mean & $1.81 \mathbf{a}$ & $1.61 \mathbf{b}$ & $1.67 \mathbf{a b}$ & \multirow{3}{*}{ NS } & 1.70 \\
\hline & SE & 0.04 & 0.01 & 0.05 & & 0.04 \\
\hline & $\mathrm{CV}$ & 3.12 & 0.43 & 4.64 & & 5.83 \\
\hline \multirow{3}{*}{11} & Mean & 2.01 & 1.90 & 1.94 & \multirow{3}{*}{$\mathrm{NS}$} & 1.95 \\
\hline & SE & 0.03 & 0.08 & 0.01 & & 0.03 \\
\hline & $\mathrm{CV}$ & 2.11 & 5.95 & 0.36 & & 3.75 \\
\hline \multirow{3}{*}{12} & Mean & 2.27 & 1.99 & 1.99 & \multirow{3}{*}{ NS } & 2.08 \\
\hline & SE & 0.07 & 0.01 & 0.09 & & 0.06 \\
\hline & $\mathrm{CV}$ & 4.66 & 0.71 & 6.39 & & 7.90 \\
\hline \multirow{3}{*}{13} & Mean & 1.98 & 1.83 & 1.89 & \multirow{3}{*}{$\mathrm{NS}$} & 1.90 \\
\hline & SE & 0.07 & 0.07 & 0.02 & & 0.03 \\
\hline & $\mathrm{CV}$ & 5.34 & 5.78 & 1.86 & & 5.06 \\
\hline \multirow{3}{*}{14} & Mean & 1.88 & 1.86 & 1.83 & \multirow{3}{*}{$\mathrm{NS}$} & 1.85 \\
\hline & SE & 0.05 & 0.01 & 0.09 & & 0.02 \\
\hline & $\mathrm{CV}$ & 3.76 & 0.76 & 7.32 & & 3.82 \\
\hline \multirow{3}{*}{15} & Mean & 2.29 & 2.22 & 2.27 & \multirow{3}{*}{$\mathrm{NS}$} & 2.26 \\
\hline & SE & 0.15 & 0.05 & 0.07 & & 0.04 \\
\hline & $\mathrm{CV}$ & 9.26 & 3.49 & 4.36 & & 5.05 \\
\hline \multirow{3}{*}{16} & Mean & 2.73 & 2.62 & 2.81 & \multirow{3}{*}{ NS } & 2.72 \\
\hline & SE & 0.15 & 0.39 & 0.01 & & 0.11 \\
\hline & $\mathrm{CV}$ & 8.01 & 21.05 & 0.25 & & 10.26 \\
\hline \multirow{3}{*}{17} & Mean & 2.87 & 2.99 & 3.05 & \multirow{3}{*}{$\mathrm{NS}$} & 2.97 \\
\hline & SE & 0.03 & 0.10 & 0.05 & & 0.04 \\
\hline & $\mathrm{CV}$ & 1.47 & 4.73 & 2.54 & & 3.78 \\
\hline \multirow{3}{*}{18} & Mean & 4.20 & 3.40 & 3.73 & \multirow{3}{*}{$\mathrm{NS}$} & 3.78 \\
\hline & SE & 0.12 & 0.46 & 0.02 & & 0.19 \\
\hline & $\mathrm{CV}$ & 4.20 & 19.31 & 0.94 & & 12.46 \\
\hline \multirow{3}{*}{19} & Mean & 4.42 & 4.36 & 4.48 & \multirow{3}{*}{$\mathrm{NS}$} & 4.42 \\
\hline & SE & 0.04 & 0.48 & 0.53 & & 0.18 \\
\hline & $\mathrm{CV}$ & 1.28 & 15.56 & 16.73 & & 10.31 \\
\hline \multirow{3}{*}{ 9-19 } & Mean & $2.53 \mathrm{a}$ & $2.38 \mathrm{~b}$ & $2.46 \mathrm{ab}$ & \multirow{3}{*}{ NS } & 2.46 \\
\hline & SE & 0.00 & 0.02 & 0.04 & & 0.03 \\
\hline & $\mathrm{CV}$ & 0.27 & 1.18 & 2.58 & & 3.09 \\
\hline
\end{tabular}

$\mathrm{a}, \mathrm{b}, \ldots$ Treatment means having different superscript litters within the same raw are significantly different at $(\mathrm{P} \leq 0.05)$ using Duncan's Multiple Range test. 
Table (6): Least square means, standard errors (S.E.) and coefficients of variation (CV\%) for slaughter parameters (\%) of BUT9 turkeys fed on diets contained 0.0 (control), $0.4 \%$ (T1) or $0.8 \%$ (T2) Origanum majorana.

\begin{tabular}{|c|c|c|c|c|c|c|c|c|c|}
\hline & & Control & T1 & $\mathbf{T 2}$ & Sig. & Male & Female & Sig & $\begin{array}{c}\text { Overall } \\
\text { mean }\end{array}$ \\
\hline \multirow{4}{*}{ Feather } & Mean & 5.80 & 5.80 & 5.44 & \multirow{3}{*}{ NS } & 5.43 & 5.98 & \multirow{3}{*}{ NS } & 5.73 \\
\hline & $\mathrm{SE}$ & 1.14 & 0.91 & 1.28 & & 0.66 & 0.93 & & 0.57 \\
\hline & $\mathrm{CV}$ & 39.18 & 35.03 & 33.26 & & 27.22 & 38.05 & & 32.85 \\
\hline & Mean & 4.49 & 4.35 & 4.27 & \multirow{3}{*}{ NS } & 4.76 & 4.07 & \multirow{3}{*}{ NS } & 4.38 \\
\hline \multirow[t]{2}{*}{ Blood } & SE & 0.25 & 0.32 & 0.56 & & 0.17 & 0.24 & & 0.18 \\
\hline & $\mathrm{CV}$ & 11.31 & 16.68 & 18.49 & & 7.86 & 14.36 & & 13.64 \\
\hline \multirow{4}{*}{ Head } & Mean & 2.04 & 1.98 & 1.93 & \multirow{3}{*}{ NS } & 2.11 & 1.90 & \multirow{3}{*}{ NS } & 1.99 \\
\hline & SE & 0.18 & 0.09 & 0.23 & & 0.09 & 0.11 & & 0.08 \\
\hline & $\mathrm{CV}$ & 17.41 & 10.52 & 17.13 & & 9.88 & 14.59 & & 13.06 \\
\hline & Mean & 0.16 & 0.10 & 0.06 & \multirow{3}{*}{ NS } & 0.11 & 0.12 & \multirow{3}{*}{ NS } & 0.11 \\
\hline \multirow[t]{2}{*}{ Abdominal Fat } & $\mathrm{SE}$ & 0.03 & 0.01 & 0.01 & & 0.03 & 0.02 & & 0.02 \\
\hline & $\mathrm{CV}$ & 38.64 & 25.51 & 32.19 & & 65.38 & 36.35 & & 48.23 \\
\hline \multirow{3}{*}{ Heart } & Mean & 0.40 & 0.42 & 0.30 & \multirow{3}{*}{ NS } & 0.38 & 0.39 & \multirow{3}{*}{ NS } & 0.39 \\
\hline & $\mathrm{SE}$ & 0.05 & 0.02 & 0.00 & & 0.04 & 0.04 & & 0.02 \\
\hline & $\mathrm{CV}$ & 27.36 & 11.44 & 1.88 & & 21.98 & 22.12 & & 20.98 \\
\hline \multirow{3}{*}{ Liver } & Mean & 1.43 & 1.40 & 1.78 & \multirow{3}{*}{ NS } & 1.38 & 1.57 & \multirow{3}{*}{ NS } & 1.48 \\
\hline & SE & 0.11 & 0.08 & 0.16 & & 0.06 & 0.11 & & 0.07 \\
\hline & $\mathrm{CV}$ & 15.09 & 12.59 & 12.90 & & 10.21 & 16.98 & & 15.64 \\
\hline \multirow{3}{*}{ Gizzard } & Mean & 1.64 & 1.63 & 1.70 & \multirow{3}{*}{ NS } & 1.56 & 1.72 & \multirow{3}{*}{ NS } & 1.65 \\
\hline & SE & 0.06 & 0.09 & 0.25 & & 0.03 & 0.09 & & 0.06 \\
\hline & $\mathrm{CV}$ & 6.80 & 12.76 & 21.08 & & 5.02 & 12.88 & & 11.30 \\
\hline \multirow{3}{*}{$\begin{array}{c}\text { Eviscerated } \\
\text { weight }\end{array}$} & Mean & 79.00 & 79.42 & 78.70 & \multirow{3}{*}{ NS } & 78.95 & 79.29 & \multirow{3}{*}{ NS } & 79.14 \\
\hline & $\mathrm{SE}$ & 1.36 & 0.49 & 0.12 & & 0.71 & 0.76 & & 0.50 \\
\hline & $\mathrm{CV}$ & 3.45 & 1.37 & 0.22 & & 2.02 & 2.35 & & 2.11 \\
\hline
\end{tabular}

Table (7): Least square means, standard errors (S.E) and coefficients of variation (CV\%) for plasma constituents of BUT9 turkeys fed on diets contained 0.0 (control), $0.4 \%$ (T1) or $0.8 \%$ (T2) Origanum majorana.

\begin{tabular}{|c|c|c|c|c|c|c|c|c|c|}
\hline & & Control & T1 & $\mathbf{T 2}$ & Sig. & Male & Female & Sig & $\begin{array}{c}\text { Overall } \\
\text { mean }\end{array}$ \\
\hline \multirow{3}{*}{$\begin{array}{l}\text { Albumin } \\
\text { (g/dl) }\end{array}$} & Mean & 0.91 & 0.95 & 0.80 & \multirow{3}{*}{ NS } & 0.87 & 0.90 & \multirow{3}{*}{ NS } & 0.89 \\
\hline & SE & 0.09 & 0.05 & 0.10 & & 0.03 & 0.10 & & 0.05 \\
\hline & $\mathrm{CV}$ & 13.99 & 7.44 & 17.68 & & 5.29 & 19.25 & & 12.89 \\
\hline \multirow{3}{*}{$\begin{array}{l}\text { Globulin } \\
\text { (g/dl) }\end{array}$} & Mean & 3.09 & 3.75 & 3.25 & \multirow{3}{*}{ NS } & 4.03 & 2.70 & \multirow{3}{*}{ NS } & 3.36 \\
\hline & SE & 1.09 & 1.25 & 0.35 & & 0.61 & 0.47 & & 0.46 \\
\hline & $\mathrm{CV}$ & 49.89 & 47.14 & 15.23 & & 26.28 & 30.32 & & 33.16 \\
\hline \multirow{3}{*}{ A/G ratio } & Mean & 34.81 & 29.00 & 25.24 & \multirow{3}{*}{ NS } & 22.88 & 36.48 & \multirow{3}{*}{ NS } & 29.68 \\
\hline & SE & 15.19 & 11.00 & 5.80 & & 4.10 & 9.00 & & 5.37 \\
\hline & $\mathrm{CV}$ & 61.71 & 53.64 & 32.48 & & 31.03 & 42.71 & & 44.28 \\
\hline \multirow{3}{*}{$\begin{array}{c}\text { Total Protein } \\
\text { (g/dl) }\end{array}$} & Mean & 4.00 & 4.70 & 4.05 & \multirow{3}{*}{ NS } & 4.90 & 3.60 & \multirow{3}{*}{ NS } & 4.25 \\
\hline & SE & 1.00 & 1.20 & 0.25 & & 0.61 & 0.38 & & 0.43 \\
\hline & $\mathrm{CV}$ & 35.36 & 36.11 & 8.73 & & 21.50 & 18.22 & & 24.93 \\
\hline \multirow{3}{*}{$\begin{array}{c}\text { ALT (GPT) } \\
(\mathbf{U} / \mathbf{L})\end{array}$} & Mean & 10.00 & 5.70 & 4.85 & \multirow{3}{*}{ NS } & 7.13 & 6.57 & \multirow{3}{*}{ NS } & 6.85 \\
\hline & SE & 1.00 & 1.00 & 0.85 & & 1.95 & 1.44 & & 1.09 \\
\hline & $\mathrm{CV}$ & 14.14 & 24.81 & 24.79 & & 47.46 & 38.11 & & 39.14 \\
\hline \multirow{3}{*}{$\begin{array}{c}\text { AST (GOT) } \\
\text { (U/L) }\end{array}$} & Mean & 117.40 & 113.30 & 111.95 & \multirow{3}{*}{ NS } & 113.30 & 115.13 & \multirow{3}{*}{ NS } & 114.22 \\
\hline & SE & 0.40 & 2.80 & 0.35 & & 2.27 & 1.44 & & 1.27 \\
\hline & $\mathrm{CV}$ & 0.48 & 3.49 & 0.44 & & 3.47 & 2.17 & & 2.73 \\
\hline \multirow{3}{*}{$\begin{array}{l}\text { Creatinine } \\
\text { (mg/dl) }\end{array}$} & Mean & 0.59 & 0.32 & 0.21 & \multirow{3}{*}{$*$} & 0.39 & 0.35 & \multirow{3}{*}{ NS } & 0.37 \\
\hline & $\mathrm{SE}$ & 0.03 & 0.04 & 0.02 & & 0.13 & 0.10 & & 0.07 \\
\hline & $\mathrm{CV}$ & 8.46 & 20.20 & 10.35 & & 55.53 & 51.31 & & 48.55 \\
\hline \multirow{3}{*}{$\begin{array}{l}\text { Triglycerides } \\
\text { (mg/dl) }\end{array}$} & Mean & 67.25 & 37.70 & 24.15 & \multirow{3}{*}{ NS } & 49.73 & 36.33 & \multirow{3}{*}{ NS } & 43.03 \\
\hline & SE & 1.75 & 22.70 & 0.85 & & 13.30 & 16.59 & & 9.97 \\
\hline & $\mathrm{CV}$ & 3.68 & 85.15 & 4.98 & & 46.31 & 79.07 & & 56.74 \\
\hline \multirow{3}{*}{$\begin{array}{c}\text { Cholesterol } \\
\text { (mg/dl) }\end{array}$} & Mean & 211.00 & 121.50 & 107.00 & \multirow{3}{*}{$*$} & 152.00 & 141.00 & \multirow{3}{*}{ NS } & 146.50 \\
\hline & SE & 15.00 & 1.50 & 3.00 & & 37.11 & 28.04 & & 20.95 \\
\hline & $\mathrm{CV}$ & 10.05 & 1.75 & 3.97 & & 42.29 & 34.45 & & 35.02 \\
\hline
\end{tabular}

$\mathrm{a}, \mathrm{b}, \ldots$ Treatment means having different superscript litters within the same raw are significantly different at $(\mathrm{P} \leq 0.05)$ using 


\section{Physical characteristics of meat}

Significant treatments effects $(\mathrm{P} \leq 0.05$ or $\mathrm{P} \leq 0.01$ or $\mathrm{P} \leq 0.0001)$ were detected on tenderness breast and thigh meat, color lightness $\left(\mathrm{L}^{*}\right)$ of thigh and breast meat, redness $\left(a^{*}\right)$ and yellowness $\left(b^{*}\right)$ of thigh only (Table 8 ). The best values of meat color measurements were generally recorded by the groups fed on treated diets containing Origanum majorana compared with control group. These values demonstrated that meat color of the BUT9 commercial turkeys fed diets containing Origanum majorana tend to give degrees from bright, redness and yellowish more than control group (i.e. improved meat quality). Also, Florou-Paneri et al. (2006) reported that improve the oxidative stability of meat of broiler fed dietary oregano compared to the control group. Badee et al. (2013) showed that marjoram oil might work as reducing agent which could reduce metmyoglobin formation to some degree and improve of the color values. Li et al. (2006) showed that the bioactive components in the aromatic plants possess the ability to protect the body from damage caused by free radicals induced oxidative stress by quenching singlet oxygen and inducing cytochrome or other enzymes and they also contain antimicrobial compounds which contribute to the retardation of microbial growth on foods especially meat products.

Table (8): Least square means, standard errors (S.E) and coefficients of variation (CV\%) for meat physical parameters of BUT9 turkeys fed on diets contained 0.0 (control), $0.4 \%$ (T1) or $0.8 \%$ (T2) Origanum majorana.

\begin{tabular}{|c|c|c|c|c|c|c|c|c|c|}
\hline & & Control & T1 & $\mathbf{T 2}$ & Sig. & Male & Female & Sig & $\begin{array}{c}\text { Overall } \\
\text { mean }\end{array}$ \\
\hline \multirow{4}{*}{ Tenderness (breast) } & Mean & $2.13^{\mathrm{a}}$ & $2.13^{b}$ & $1.63^{\mathrm{c}}$ & \multirow{3}{*}{$* * * *$} & 2.08 & 1.83 & \multirow{3}{*}{$* * * *$} & 1.96 \\
\hline & SE & 0.13 & 0.13 & 0.13 & & 0.17 & 0.17 & & 0.12 \\
\hline & $\mathrm{CV}$ & 8.32 & 8.32 & 10.88 & & 13.86 & 15.75 & & 14.92 \\
\hline & Mean & $2.63^{\mathrm{a}}$ & $2.50^{\mathrm{a}}$ & $2.00^{\mathbf{b}}$ & \multirow{3}{*}{$*$} & 2.42 & 2.33 & \multirow{3}{*}{ NS } & 2.38 \\
\hline \multirow[t]{2}{*}{ Tenderness (thigh) } & SE & 0.13 & 0.00 & 0.00 & & 0.22 & 0.17 & & 0.13 \\
\hline & $\mathrm{CV}$ & 6.73 & 0.00 & 0.00 & & 15.80 & 12.37 & & 12.89 \\
\hline \multirow{3}{*}{ W.H.C (breast) } & Mean & 39.10 & 45.75 & 47.60 & \multirow{3}{*}{ NS } & 41.80 & 46.50 & \multirow{3}{*}{ NS } & 44.15 \\
\hline & SE & 1.00 & 5.55 & 0.50 & & 2.72 & 3.33 & & 2.19 \\
\hline & $\mathrm{CV}$ & 3.62 & 17.16 & 1.49 & & 11.26 & 12.41 & & 12.16 \\
\hline \multirow{3}{*}{ W.H.C (thigh) } & Mean & 40.85 & 45.05 & 48.30 & \multirow{3}{*}{ NS } & 43.80 & 45.67 & \multirow{3}{*}{ NS } & 44.73 \\
\hline & SE & 0.05 & 3.95 & 1.10 & & 2.80 & 2.49 & & 1.73 \\
\hline & $\mathrm{CV}$ & 0.17 & 12.40 & 3.22 & & 11.07 & 9.44 & & 9.45 \\
\hline \multirow{3}{*}{ Breast pH } & Mean & 5.77 & 5.60 & 5.48 & \multirow{3}{*}{ NS } & 5.68 & 5.55 & \multirow{3}{*}{ NS } & 5.62 \\
\hline & SE & 0.03 & 0.17 & 0.01 & & 0.10 & 0.09 & & 0.07 \\
\hline & $\mathrm{CV}$ & 0.74 & 4.17 & 0.26 & & 3.17 & 2.96 & & 3.00 \\
\hline \multirow{3}{*}{ Thigh pH } & Mean & 5.71 & 5.55 & 5.41 & \multirow{3}{*}{ NS } & 5.60 & 5.51 & \multirow{3}{*}{ NS } & 5.56 \\
\hline & SE & 0.01 & 0.15 & 0.02 & & 0.11 & 0.10 & & 0.07 \\
\hline & $\mathrm{CV}$ & 0.25 & 3.82 & 0.52 & & 3.30 & 3.00 & & 2.97 \\
\hline \multirow{4}{*}{ Uric acid in meat } & Mean & $0.46^{\mathrm{a}}$ & $0.40^{\mathbf{a b}}$ & $0.33^{\mathbf{b}}$ & \multirow{3}{*}{ NS } & 0.41 & 0.38 & \multirow{3}{*}{ NS } & 0.39 \\
\hline & SE & 0.01 & 0.03 & 0.03 & & 0.03 & 0.05 & & 0.03 \\
\hline & $\mathrm{CV}$ & 1.55 & 10.61 & 10.88 & & 12.91 & 21.29 & & 16.13 \\
\hline & Mean & $2.75^{\mathrm{a}}$ & $2.15^{\mathrm{ab}}$ & $1.70^{\mathbf{b}}$ & & 2.17 & 2.23 & & 2.20 \\
\hline Uric acid in liver & SE & 0.15 & 0.15 & 0.10 & NS & 0.38 & 0.23 & NS & 0.20 \\
\hline & $\mathrm{CV}$ & 7.71 & 9.87 & 8.32 & & 30.73 & 18.10 & & 22.45 \\
\hline & Mean & 3.75 & 3.53 & 3.35 & & 3.70 & 3.38 & & 3.54 \\
\hline Uric acid in blood & SE & 0.05 & 0.13 & 0.30 & NS & 0.05 & 0.19 & NS & 0.11 \\
\hline & $\mathrm{CV}$ & 1.89 & 5.01 & 12.66 & & 2.34 & 9.62 & & 7.75 \\
\hline & Mean & $64.86^{\mathrm{a}}$ & $62.17^{b}$ & $67.00^{\mathrm{a}}$ & & 64.36 & 65.16 & & 64.76 \\
\hline Lightness (thigh) & $\mathrm{SE}$ & 1.16 & 0.73 & 1.18 & $* *$ & 1.03 & 0.98 & NS & 0.70 \\
\hline & $\mathrm{CV}$ & 5.36 & 3.53 & 5.56 & & 5.96 & 5.63 & & 5.72 \\
\hline Lightness (breast) & Mean & $66.83^{\mathrm{a}}$ & $64.56^{b}$ & $67.29^{\mathrm{a}}$ & & 64.68 & 67.85 & $* * *$ & 66.26 \\
\hline & SE & 1.00 & 1.99 & 0.51 & $* *$ & 1.26 & 0.57 & & 0.75 \\
\hline & $\mathrm{CV}$ & 4.49 & 9.23 & 2.41 & & 7.30 & 3.16 & & 5.95 \\
\hline & Mean & $4.44 b$ & $5.30 \mathrm{ab}$ & $6.44 a$ & & 5.55 & 5.31 & & 5.43 \\
\hline Redness (thigh) & $\mathrm{SE}$ & 0.38 & 0.15 & 0.75 & $*$ & 0.63 & 0.21 & NS & 0.33 \\
\hline & $\mathrm{CV}$ & 25.53 & 8.22 & 36.75 & & 42.73 & 14.59 & & 31.95 \\
\hline D J & Mean & $3.81^{b}$ & $3.98^{\mathrm{b}}$ & $5.36^{\mathrm{a}}$ & & 4.05 & 4.79 & & 4.42 \\
\hline Redness (breast) & SE & 0.50 & 0.22 & 0.63 & NS & 0.44 & 0.42 & NS & 0.31 \\
\hline & $\mathrm{CV}$ & 39.61 & 16.47 & 37.35 & & 40.64 & 33.12 & & 36.88 \\
\hline & Mean & $4.08^{b}$ & $3.51^{\mathrm{b}}$ & $4.98^{\mathrm{a}}$ & & 4.00 & 4.44 & & 4.22 \\
\hline Yellowness (thigh) & SE & 0.51 & 0.20 & 0.20 & $* *$ & 0.32 & 0.30 & NS & 0.22 \\
\hline & $\mathrm{CV}$ & 37.67 & 17.41 & 12.80 & & 29.58 & 25.38 & & 27.38 \\
\hline & Mean & $4.81^{b}$ & $6.39^{\mathrm{ab}}$ & $7.12^{\mathrm{a}}$ & & 4.80 & 7.49 & & 6.14 \\
\hline Yellowness (breast) & SE & 0.70 & 0.67 & 0.99 & NS & 0.65 & 0.55 & $* *$ & 0.49 \\
\hline & $\mathrm{CV}$ & 43.43 & 31.66 & 43.87 & & 50.64 & 27.29 & & 42.21 \\
\hline
\end{tabular}

$\mathrm{a}, \mathrm{b}, \ldots$ Treatment means having different superscript litters within the same raw are significantly different at $(\mathrm{P} \leq 0.05)$ using

Duncan's Multiple Range test. 
The control group gave the highest values of thigh and breast $\mathrm{pH}$, thigh and breast tenderness, uric acid in blood, liver and meat except that for water holding capacity compared with two Origanum majorana treatments. Teixeira et al. (2013) reported that the lower $\mathrm{pH}$ values reflect antimicrobial properties of marjoram oil which reduces the accumulation of basic substances. Cornforth (1994) stated that meat with high $\mathrm{pH}$ has a higher water binding capacity, hence making it appear darker. Moreover, Kauffman and Marsh (1987) and Cornforth (1994) reported that when the $\mathrm{pH}$ of the meat is above the iso-electric point of the myofibril proteins in the meat, water molecules are tightly bound, and the meat appears darker in color. El-Sharkawy (1984) showed that meat containing a relatively higher protein is capable to hold much water than those of a lower protein containing meat.

The results proved significant sex effects on breast meat tenderness, lightness and yellowness breast color. These values demonstrated that meat color of females tend to give degrees of lightness, redness and yellowish better than that of males. El-Naggar et al. (2000) reported that most sex differences were not significantly detectable for most meat physical characteristics at various age stages. However, Smith et al. (2015) showed that sex affect breast meat quality of duck and the values of lightness and yellowness were higher in male than female. The least square means showed that males recorded higher values of $\mathrm{pH}$, uric acid in blood, liver as well as tenderness of thigh and breast meat while the females gave the highest WHC of thigh and breast values. Hartman et al. (2006) reported that the concentration of plasma uric acid in birds was high in both males and females chickens and levels in males were relatively higher at evaluated ages.

These results provided evidences that diets containing Origanum majorana had no adverse and may have beneficial effects on turkey meat and did not restrict the use of turkey meat for further processing and product added value. Also, it seemed that females' meat quality is rather better in its show performance than that of males.

\section{CONCLUSION}

It can be concluded that supplementing of Origanum majorana to basal diet improved growth performance, meat quality. Also, no adverse and restrict effects of using turkey meat.

\section{REFERENCES}

Abdel-Azeem, F. (2002). Digestion, neomycin and yeast supplementation in broiler diets under Egyptian summer conditions. Egypt Poult. Sci., 22: 235-257.

Abdo, Z.M.A., A.Z.M. Soliman and O.S. Barakat (2003). Effect of hot pepper and marjoram as feed additives on the growth performance and the microbial population of the gastrointestinal tract of broilers. Egypt Poult. sci., 23: 91-113.

Al-Harthi, M.A. (2002a). Efficacy of vegetable diets with antibiotics and different types of spices or their mixtures on performance, economic efficiency and carcass traits of broilers. J. Agric. Sci. Mansoura, Univ., 27: 3531-3545.

Al-Harthi, M.A. (2002b). Performance and carcass characteristics of broiler chicks as affected by different dietary types and levels of herbs and spices as no classical growth promoters. Egypt Poult. Sci., 22: 325-343.

Al-Harthi, M.A. (2004). Efficiency of utilizing some spices and herbs with or without antibiotic supplementation on growth performance and carcass characteristics of broiler chicks. Egypt Poult. Sci., 24: 869-899.

Ali, A.H.H. (2014). Productive performance and immune response of broiler chicks as affected by dietary marjoram leaves powder. Egypt Poult. Sci. 34 (I): 57-70.

Al-Shuwaili, M.A., I.E. Ibrahim and M.T.N. Al-Bayati (2015). Effect of dietary herbal plants supplement in turkey diet on performance and some blood biochemical parameters. Global journal of bioscience and biotechnology.4 (2): 153-157.

Ayala, L., N. Silvana, I. Zocarrato and S. Gomez (2011). Use of vulgar oregano (Origanum vulgare) as phytobiotic in fatting rabbits. Cuban J. Agric. Sci. 45: 159-161.

Azouz, H.M.M. (2001). Effect of hot pepper and fenugreek seeds supplementation on broiler diets. Ph.D. Thesis, Faculty of Agric., Cairo University, Egypt.

Badee, A.Z.M., R.K. Moawad, M.M. El-Noketi and M.M. Gouda. (2013). Antioxidant and antimicrobial activities of marjoram (Origanum majorana L.) essential oil. Journal of Applied Sciences Research, 9(2): 1193-1201.

Bampidis, V.A., V. Christodoulou, P. Florou-Paneri, E. Christaki, P.S. Chatzopoulou, T. Tsiligianni and A.B. Spais (2005). Effect of dietary dried oregano leaves on growth performance, carcase characteristics and serum cholesterol of female early maturing turkeys. British poultry science, 46 : 595-601.

Bartels, H., M. Böhmer and C. Heierli (1972). Serum kreatininbestimmung ohne enteweissen. Clinica Chimica Acta, 37: 193-197.

Bernd, R.E. and G.G. George (1977). Fluorometric estimation of triglycerides in serum by a modification of method of Bucolo and David. CLIN. CHEM, 23: 286-288.

C.1.E. (1978). Recommendation on uniform color spaces, color difference equations, psychometric color terms. Supplement No. 2 to C.I.E. publication No. 15 (E-1.3.1) 1971/(TC-1.3) 1978. Bureau Central de la C.I.E., Paris, France.

Cornforth, D.P. (1994). Color and its importance. Pages 34-78 in: Quality attributes and their measurements in meat, poultry and fish products. A. M. Pearson and T. R. Dutson, ed. Chapman and Hall London, UK.

Cowan, M.M. (1999). Plant products as antimicrobial agents. Clinical Microbiology Reviews, 12, 4: 564582. 
Craig, W.J. (1999). Health-promoting properties of common herbs. American Journal of Clinical Nutrition, 70: 491S-499S.

Del Campo, J., M.J. Amiot and C. Nguyen (2000). Antimicrobial effect of rosemary extract. J. food protection. 63:1359-1368.

Dina El Bushuty, H. and Naglaa M. Shanshan (2012). Effect of natural herbs of marjoram And ginger on hypercholesterolemic Rats. The Annual conference (Seventh Arabic - Fourth International), 17541767. Managing Knowledge and intellectual capital in Higher Education institutions in Egypt and Arab World. 11-12 April 2012, Faculty of Specific Education - Mansoura University.

Doumas, B., W.A. Watson and H.G. Biggs (1971). Albumin standards and measurements of serum albumin with bromocresol green. Clin. Chim. Acta, 31: 87-96.

Duncan, D.B. (1955). The Multiple Range and Multiple F Test. Biometrics, 11: 1-42.

EL-Ashmawy, I.M., A.F. EL-Nahas and O.M. Salama (2005). Protective effect of volatile oil, alcoholic and aqueous extract of Origanum majorana on lead acetate toxicity in mice. Basic Clin Pharmacol Toxicol., 97(4): 238-243.

El-Kaiaty, A.M., A.Z.M. Soliman and M.S.H. Hassan (2002a). Combined effect of garlic, fenugreek and black seed on some productive and physiological responses of laying hens. Egypt Poult. Sci., 22: 147-174

El-Kaiaty, A.M., A.Z.M. Soliman and M.S.H. Hassan (2002b). The physiological and immunological effects of some natural feed additives in layer hen diets. Egypt Poult. Sci, 22: 175-203.

EL-Mallah, G.M., Sh. A.M. Ibrahim and Zeinab M.A. Abdo (2005). Garlic and fenugreek as feed additives to different levels of protein and energy in diets of growing turkeys. Egypt Poult. Sci., 25: 911- 929.

El-Naggar, N.M., A.M. Abdel-Ghany, Y.A. Dowidar and E.M.A. Qota (2000). Influence of crossing white holland and broad breasted bronze turkeys on meat and serum characteristics. Egypt Poult. Sci., 20: 441- 465.

El-Sharkawy, A.M. (1984). Chemical and technological studies on meats. M.Sc. Thesis Fac. Agric. Tanta Univ. Egypt.

Emam, R.M.S. (2006). Productive performance of Japanese quails as affected by feeding on some medicinal and aromatic plants and their byproducts with or without enzymes supplementions. M.Sc. Thesis. Faculty of Agriculture, Fayoum University.

Ersoy, I.E. and K. Çelik (2007). Effects of the whole grain, Saccharomyces cerevisiae and yoghurt on performance and some organ characteristics in Turkeys. Archiva Zootechnica, 10: 33-39.

Florou-Paneri, P., I. Giannenas, E. Christaki, A. Govaris and N. Botsoglu (2006). Performance of chickens and oxidative stability of the produced meat as affected by feed supplementation with oregano, vitamin $\mathrm{C}$, vitamin $\mathrm{E}$ and their combinations. Arch. Geflügelk., 70(5): 232- 240.
Ghazalah, A.A., O.M. El-Husseiny, H.M. Fayek and S. Abou El-Waffa (1994). Influence of enzyme preparation and growth prometer on broiler performance. The $2^{\text {nd }}$ Scci. Conf. of Poult. Kafr ElSheikh, Egypt.

Giannenas, I., P. Florou-Paneri, N.A. Botsoglou, E. Christaki and A.B. Spais (2005). Effect of feed supplementation with dehydrated oregano plants on the performance of broiler chickens and the oxidative stability of the produced meat. J. Animal Feed Sci. 14: 521-535.

Guo, F.C. (2003). Mushroom and herb polysaccharides as alternative for antimicrobial growth promoters on poultry. Thesis, Wageningen Institute of Animal Sciences, Department of Animal Nutrition, Wageningen University, Wageningen, Netherlands.

Hallnet, Ltd. (2015). http://www.herbwisdom.com/herbmarjoram.html.

Hancock, C.B., G.D. Breadform and G.C. Bmmans (1994). Potential growth of males and females of commercials broilers. British Poultry Science, 73: 247-264.

Hartman, S., S.A. Taleb, T. Geng, K. Gyenai, X Guan and E. Smith (2006). Comparison of plasma uric acid levels in five varieties of the domestic Turkey, Meleagris gallopavo. Poultry Science 85: 17911794.

Heinonen, I.M., P.J. Lehtonen and A.I. Hopia (1998). Anti oxidant activity of berry and fruit wines and liquors. J. Agric. Food Chem., 46: 25-31.

Hermann, M. (1973). Herbs and medicinal flowers. Galahed book, New Yok.

Hsieh, P.C., J.L. Mau and S.H. Huang (2001). Antimicrobial effect of various combinations of plant extracts. Food Microbiol.. 18: 35-43.

Isguzar, E. (2003). Growth, carcass traits and meat quality of Bronze and White Turkeys in Isparta province of Turkey. Arch. Tierz., Dummerstorf, 46(5): 471-481.

Jauregui, C.A., J.M. Regenstein and R.C. Baker (1981). A simple centrifugal method for measuring expressible moisture, a water-binding property of muscle foods. J. Food Sci., 46: 1271-1273.

Jeacocke, R.E. (1977). Continuous measurements of the $\mathrm{pH}$ of beef muscle in intact beef carcasses. J. Food Technol., 12: 375-386.

Kageyama, N.A. (1971). Direct colorimetric determination of uric acid in serum and urine with uricase-catalase system. Clin. Chim. Acta, 31: 421-426.

Kauffman, R.G., and B.B. Marsh (1987). Quality Characteristics of Muscle as Food. Pages 356-357 in: The Science of Meat and Meat Products. 3rd ed. J.F. Price and B.S. Schweigert, ed. Food and Nutrition Press, Inc., Westport, CT.

Leeja, L. and J.E. Thoppil (2007). Antimicrobial activity of methanol extract of Origanum majorana L. (Sweet marjoram). Journal of Environmental Biology, 28(1): 145-146.

Li, T.S.C. (2006) The range of medicinal herbs and spices. In Handbook of Herbs and Spices; Peter, 
K.V., Ed.; Woodhead Publishing Limited: Cambridge, UK, 3: 113-125.

Mona Osman, H. M. Yakout, Motawe, H. F. and Ezz El-Arab, W. F. (2010). Productive, physiological, immunological and economical effects of supplementing natural feed additives to broiler diets. Egypt. Poult. Sci. 30 (1): 25-53

Mostafa, M.Y. and R.Y. Nofal (2000). Effects of crossing two breeds of turkey on live body measurements, growth performance and livability. Egypt Poult. Sci., 20: 239-252.

Nagm, D.R. (2002). Effect of some common herbs on weight reduction in obese rats. M.S.C. Thesis, Faculty of Home Economics, Minufiya University.

National Research Council, NRC (1994). Nutritional requirement of poultry $9^{\text {th }}$ rev. ed. National Academy Press, Washington, D.C.USA.

Ogundu Uduak, E., V.M.O. Okoro, G.U. Okeke, N. Durugo, G.A.C. Mbaebie and C.I. Ezebuike (2013). Effects of age, breed and sex on the serum biochemical values of Turkeys (Meleagridis gallopova) in South-eastern Nigeria. Afr. J. Agric. Res., 8(23): 2825-2828.

Osman, M., Kh. Amber and M.A. Mahmoud (2004). Response of broiler chicks performance to partial dietary inclusion of radish, rocket and parsley cakes. Egypt Poult. Sci., 24: 429-446.

Parry, J.W. (1969). Spices, Vol.1. Chemical Pub. Co. Brooklyn, New York. Cited from Handbook of herbs and spices. vol 1.2001, Woodhead publishing limited.

Rang, H.P. and M.M. Dale (1991). Pharmacology, $2^{\text {nd }}$ Edn., International Journal of Poultry Science, 9(10): 944-947.

Richmond, W. (1973). Preparation and properties of a cholesterol oxidase from Nocardia sp. and its application to the enzymatic assay of total cholesterol in serum. CLIN. CHEM., 19/12, 13501356.

Rodriguez-Meizoso, I., F.R. Marin, M. Herrero, F.J. Senorans, G. Reglero, A. Cifuentes and E. Ibanez (2006). Subcritical water extraction of nutraceuticals with antioxidant activity from oregano: Chemical and functional characterization. J. Pharm Biomed Anal, 41(5): 1560-1565.

SAS Institute, Inc. (1998). SAS User's guide: Statistics. SAS Inst. Inc., Cary, NC. USA.

Seleem, T.S.T, H. Ibrahim, A.M. Ali, A.M. AbdElmotaal and A.E.B. Zeidan (2007). Rabbit performance as affected by Origanum majorana in diets. The $5^{\text {th }}$ Inter. Conf. on Rabbit Prod. in Hot Clim., Hurghada, Egypt, 495-508.

Shamseldin, R. M., S. Gibril, M. Atta, O.E. Yassin and A.A. Hassan (2014). Effect of rearing system, slaughter age and sex on turkey (Meleagris gallopavo) carcass components percentages. Res. Opin. Anim. Vet. Sci., 4(4): 198-202.
Smith, D.P., J.K. Northcutt, C.S. Sigmon and M.A. Parisi (2015). Effect of sex, bird size and marination on duck breast meat quality. International Journal of Poultry Science, 14(4): 191-195.

Soliman, A.Z., N.Y. Abd El-Malak and A.M. Abbas (1995). Effect of using some commercial feed additives as growth promoters on the performance of growing and adult rabbits. Egyptian J. Appl. Sci., 10: 501-513.

Soliman, A.Z.M., A.A. Ghazalah, S.H. El-Samra, A.M. Atta and Z.M.A. Abdo (1999). The synergistic effect of either black seed or garlic with fat on broiler performance and immunity. Egyptian J. Nutrition and Feeds 2 (Special Issue): 603-620.

Soliman, A.Z.M., M.A. Ali and Z.M.A. Abdo (2003). Effect of marjoram, bacitracin and active yeast as feed additives on the performance and the microbial content of the broiler's intestinal tract. Egypt Poult. Sci., 23: 445-467.

Tainter, D.R. and A.T. Grenis (1993) Spices and Seasonings, NCH Pub., New York. Cited from Handbook of herbs and spices. vol 1.2001 Woodhead publishing limited.

Teixeiraa, B., A. Marquesa, C. Ramosa, N.R. Nengc, J.M.F. Nogueirac, J.A. Saraivab and M.L. Nunesa, (2013). Chemical composition and antibacterial and antioxidant properties of commercial essential oils. Industrial Crops and Products, 43: 587-595.

Tietz, N.W. (1995). Clinical Guide to Laboratory Tests, $3^{\text {rd }}$ ed. Philadelphia, PA: W. B. Saunders, pp. 76.

Tiziana, B.M. and D.H.J. Dorman (1998). Antimicrobial and antioxidant properties of some commercial oleoresin. Flavour and Fragrance J, 13: 235-44.

Tollba A.A.H., M.A. Abd El-Galyl and M.H. Abd ElSamad (2005). The effect of using some herbal additives on physiological and productive performance of two Egyptian chicken strains during winter and summer seasons. Egypt Poult. Sci., 25(1): 107-123.

Triantaphyllou, K., G. Blekas and D. Boskou (2001). Ant oxidative properties of water extracts obtained from herbs of the species Lamiaceae. International-Journal-of-Food-Sciences-and Nutrition, 52(4): 313-317.

Weichselbaum, T.E. (1946). An accurate and rapid method for determination of protein in small amounts of blood serum and plasma. Am. J. Clin. Pathol., 16: 40-43.

Yadava, R.N. and V.K. Saini (1991). Antimicrobial efficacy of essential oils of Majorana hortensis Moench and Anisomeles indica (L.) Kunteze', Indian Perfumer, 35: 58-60.

Zaky, H.I and E.M. Amin (2007). Estimates of genetic parameters for body weight and body measurements in bronze turkeys (baladi) by using animal model. Egypt Poult. Sci., 27(I): 151-164. 
تأثير إضافة البردقوش على الأداء الإنتاجي، صفات الام وجودة اللحم للرومى التجاري "BUT9"

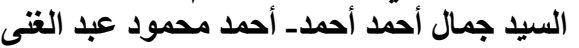

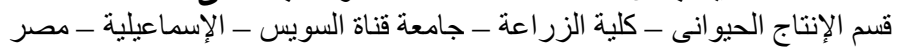

أجريت هذه الدر اسة لتقييم تأثير إضافة نبات البردقوش على التى الأداء الإنتاجي، مو اصفات الذبيحة، صفات الدم وجودة اللحم لطيور الرومي

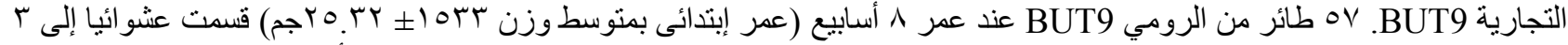

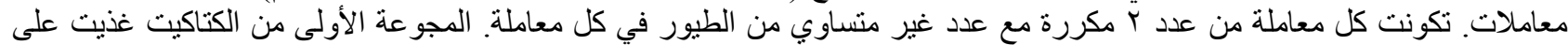

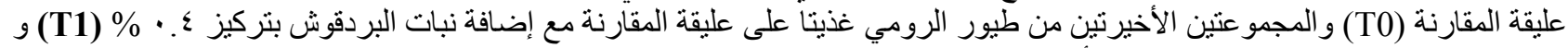

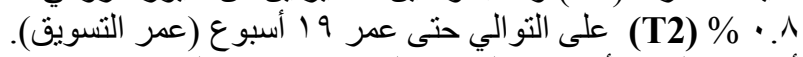

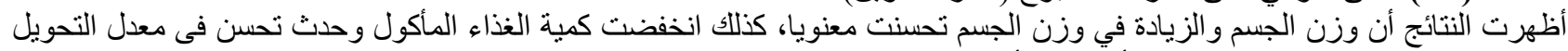

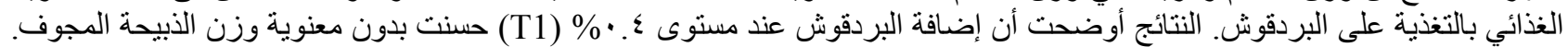

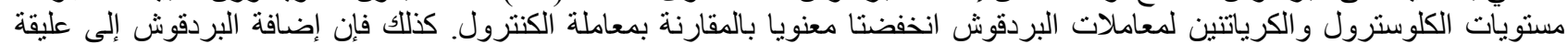

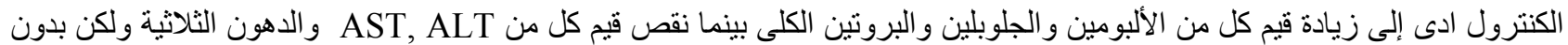
تأثير معنوي.

أظهرت النتائج وجود تأثير معنوي للمعاملات على صفات الطر اوة للحم الصدر، سطوع اللون للحم الصدر و الفخذ، و إحمرار و اصففرار اللون

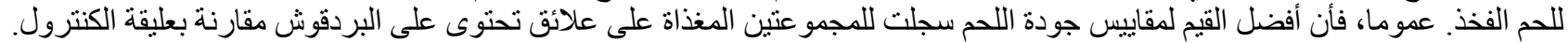

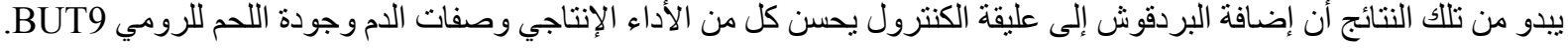

